\title{
Correction to: Cost-consequence analysis of fluticasone furoate/ vilanterol for asthma management in Spain: an analysis based on the Salford Lung Study in asthma
}

\author{
Laura Amanda Vallejo-Aparicio ${ }^{1}$. Jesús Molina ${ }^{2} \cdot$ Iñigo Ojanguren ${ }^{3,4}$ - Ana Viejo Casas $^{5}$ - Alicia Huerta ${ }^{1}$ \\ Henrik Svedsater ${ }^{6}$
}

Published online: 4 December 2019

(c) Springer-Verlag GmbH Germany, part of Springer Nature 2019

\section{Correction to: The European Journal of Health Economics https://doi.org/10.1007/s10198-019-01101-x}

In the published article, the Table 1 was published incorrectly.

The correct Table 1 is given below

\begin{tabular}{|c|c|c|}
\hline Variables & $\begin{array}{l}\text { FF/VI } \\
184 / 22 \mathrm{mcg} \\
\text { and } 92 / 22 \\
\mathrm{mcg}\end{array}$ & UC group \\
\hline Monthly drug costs $[17,21]$ & $€ 51.52^{\mathrm{a}}$ & $\begin{array}{l}\mathrm{ICS}=€ 18.75^{\mathrm{b}} \\
\mathrm{ICS} / \mathrm{LABA}=€ 48.25^{\mathrm{b}}\end{array}$ \\
\hline Annual drug costs $(€, 2018)$ & $€ 627.26$ & $€ 557.31$ \\
\hline \multicolumn{3}{|c|}{ Asthma management direct annual costs $(€, 2018)^{\mathrm{c}}[19,20]$} \\
\hline $\begin{array}{l}\text { Uncontrolled }(\mathrm{ACT} \\
\text { score } \leq 15)\end{array}$ & $€ 1919.01$ & \\
\hline $\begin{array}{l}\text { Partially controlled (ACT } \\
\text { score 16-19) }\end{array}$ & $€ 1015.61$ & \\
\hline $\begin{array}{l}\text { Well-controlled (ACT } \\
\text { score } \geq 20 \text { ) }\end{array}$ & $€ 656.90$ & \\
\hline
\end{tabular}

The original article can be found online at https://doi.org/10.1007/ s10198-019-01101-x

Laura Amanda Vallejo-Aparicio

laura.a.vallejo@gsk.com

Market Access, GSK (GlaxoSmithKline), Madrid, Spain

2 Centro de Salud Francia, Fuenlabrada, Madrid, Spain

3 Servicio de Neumología, Hospital Universitario Vall d'Hebron, Barcelona, Spain

4 Centro de Investigación en Red de Enfermedades Respiratorias (CIBERES), Instituto de Salud Carlos III (ISCIII), Barcelona, Spain

5 Centro de Salud Pisueña Cayon, Cantabria, Spain

6 Value Evidence and Outcomes, GSK, Brentford, UK

\begin{tabular}{lll}
\hline Variables & $\begin{array}{l}\text { FF/VI } \\
184 / 22 \mathrm{mcg} \\
\text { and 92/22 } \\
\text { mcg }\end{array}$ & UC group \\
& 100 & 100 \\
\hline $\begin{array}{l}\text { Assumed proportion of days } \\
\text { covered, } \%\end{array}$ & \\
Assumed current uptake, $\%$ & 10 & 90 \\
Assumed new uptake, $\%$ & 20 & 80 \\
Percentage of subjects in each ACT category & (ITT population, \\
$n=4233)$ [18] & & \\
Baseline & & \\
Uncontrolled & $41 \%$ & $41 \%$ \\
Partially controlled & $31 \%$ & $31 \%$ \\
Well-controlled & $28 \%$ & $28 \%$ \\
24 weeks & & \\
Uncontrolled & $20 \%$ & $29 \%$ \\
Partially controlled & $20 \%$ & $25 \%$ \\
Well-controlled & $60 \%$ & $46 \%$ \\
52 weeks & & \\
Uncontrolled & $21 \%$ & $30 \%$ \\
Partially controlled & $20 \%$ & $26 \%$ \\
Well-controlled & $59 \%$ & $44 \%$ \\
\hline
\end{tabular}

FF/VI fluticasone furoate/vilanterol, ICS inhaled corticosteroid, ICS/ $L A B A$ inhaled corticosteroid/long-acting beta-agonist, ACT Asthma Control Test, ITT intention-to-treat

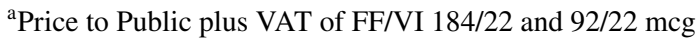

${ }^{\mathrm{b}} \mathrm{UC}$ cost calculated as a weighted average drug cost of all available presentations at Price to Public plus VAT

${ }^{\mathrm{c}}$ Costs annualised at updated to 2018 